\title{
Entrenched time delays versus accelerating opinion dynamics: are advanced democracies inherently unstable?
}

\author{
Claudius Gros ${ }^{\mathrm{a}}$ \\ Institute for Theoretical Physics, Goethe University Frankfurt, Frankfurt am Main, Germany
}

Received 14 June 2017 / Received in final form 14 September 2017

Published online 15 November 2017

(C) The Author(s) 2017. This article is published with open access at Springerlink.com

\begin{abstract}
Modern societies face the challenge that the time scale of opinion formation is continuously accelerating in contrast to the time scale of political decision making. With the latter remaining of the order of the election cycle we examine here the case that the political state of a society is determined by the continuously evolving values of the electorate. Given this assumption we show that the time lags inherent in the election cycle will inevitable lead to political instabilities for advanced democracies characterized both by an accelerating pace of opinion dynamics and by high sensibilities (political correctness) to deviations from mainstream values. Our result is based on the observation that dynamical systems become generically unstable whenever time delays become comparable to the time it takes to adapt to the steady state. The time needed to recover from external shocks grows in addition dramatically close to the transition. Our estimates for the order of magnitude of the involved time scales indicate that socio-political instabilities may develop once the aggregate time scale for the evolution of the political values of the electorate falls below 7-15 months.
\end{abstract}

\section{Introduction}

A dynamical system with time delays reacts not only to its current state, but also to what occurred in the past. It is well known in this context that time-delayed dynamical systems are prone to instabilities whenever the delay times become comparable to the time scales needed to react to current events and perturbations $[1,2]$. To give an example from economy, consider just-in-time (JIT) manufacturing, for which the time scales regulating the delivery process are typically of the order of hours [3]. Even small perturbations in the supply chain would lead to an immediate break down of JIT manufacturing, as a whole, if the management of the involved companies would need days or weeks to react to an outage.

The dynamics of democratic political systems shares certain basic similarities to manufacturing processes like just-in-time manufacturing, with the political institutions (parliament, government) reacting to shifts in the demands of the electorate [4]. It has been noticed in particular that the temporalities of economy and culture are driven by every faster cycles of innovation, change and replacement [5], with political time remaining on the other side high [6]. There is hence an evolving mismatch of the speed of formal democracy [7] with regard to the accelerating speed of capital [8], of economic

\footnotetext{
${ }^{a}$ e-mail: gros07@itp.uni-frankfurt.de
}

decision making, opinion dynamics [9] and of modern life in general [10].

The ongoing differentiation of societal time scales, with opinion dynamics accelerating in contrast to institutional decision making, did manifest itself in several political developments occurring in 2016/2017. In French politics, to give an example, electorate values changed so fast that the "En Marche" movement could raise in essentially a single year from nowhere to a center role in French politics [11]. The extended time scale of three or more years, as presently envisioned, to carry out the 2016 popular vote in favor of a Brexit [12], is on the other side exemplary for the prolonged time political institutions need to react to demands of the electorate. Our aim is here to develop a framework describing conflicts of temporalities on a basis that abstracts from specific circumstances. Our approach is particularly well suited for advanced democracies, i.e. for societies in an advanced state of acceleration.

Modern democracies are characterized additionally both by an increasing level of skepticism toward political institutions [13] and by the ongoing refinement of political correctness norms $[14,15]$. This continuously increasing sensitivity to deviations from the mainstream normative order has its equivalent in economics, where companies not adhering to normative standards of reliability will if find difficult, in a world dominated by JIT manufacturing, to build up profitable business relations. Here we show that fine-tuned political correctness norms are directly related to the underlying acceleration of societal responses. Fast 
opinion dynamics and a high level of political correctness are in our model both indicative of political systems close to a dynamical instability. Fine-tuning a political system reduces consequently its robustness against perturbations.

\section{Model}

We denote with $D=D(t)$ and with $V=V(t)$ aggregate variables measuring the state of the democracy and of the values of the electorate, its cultural dimension [16]. The time $t$ will be measured in years. We remain here on a relative abstract level, noting however that standard country-specific indicators $[17,18]$ for both democracy and values may be taken as proxies for $D$ and $V$. Alternatively one may consider the level of economic development, instead of the cultural dimension, as the basic variable interacting with the state of the democracy [19].

A political system is democratic, per definition, whenever $D(t)$ is reactive to changes in the values $V(t)$ of the electorate. This relation is captured by

$$
T_{D} \frac{d}{d t} D(t)=V(t-T)-D(t)
$$

where $T_{D}$ denotes the time democratic institutions need to aligns themselves to the demands expressed by the electorate. There is however an additional time scale involved, the time lag $T$. Time lags arise on one side from the circumstance that the electorate has to wait in a representative democracy on the average several years before it can express its views forcefully at election time [6]. Time lags also occur generically in political decision making. It will take about three years, if at all, to implement popular will in the case of the Brexit [20].

The overall process modeled by (1) describes a highly idealized democracy. We note, however, that the intricacies of real-life political decision making will enhance the effect here studied.

For the time evolution of the value $V$ we propose

$$
T_{V} \frac{d}{d t} V(t)=\sigma(D(t))-V(t)
$$

which describes a competition between a trend toward democracy $\sim \sigma(D(t))$ and an intrinsic decay term of the democratic values $\sim(-V(t))$. It has been observed in this regard that support for democratic values declines steadily in western societies [21]. If asked, to give an example, whether it is essential to live in a country that is governed democratically, over $70 \%$ of US-citizens born around 1930 would respond yes, but only about $30 \%$ of those born 1980 or later [21]. This downward trend translates in (2) to a decay time $T_{V} \approx 15-20$ years.

The actual shape of the function $\sigma(D)$ entering (2) is not relevant for the following arguments, as long as it is monotonically declining and hence reflecting that the desire to further increases the current amount $D(t)$ of democratic participation declines with its actual level. A monotonically declining $\sigma(D)$ incorporates therefore the notion of diminishing returns, which can be traced back in turn to the logarithmic discounting performed by the

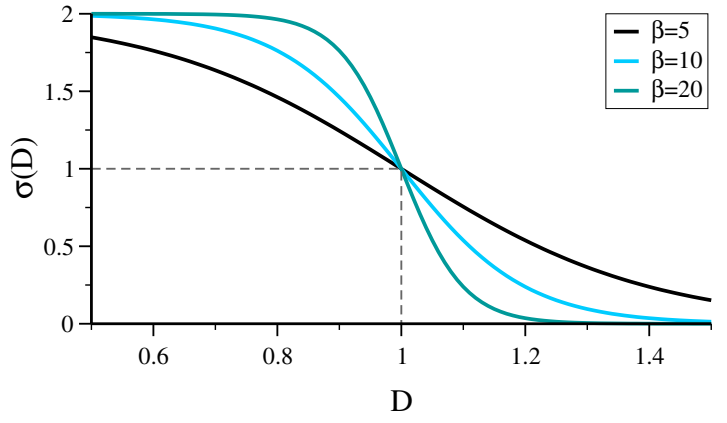

Fig. 1. The rescaled Fermi function (3) entering the evolution (2) of the values $V$ of the electorate. The monotonic decline of $\sigma(D)$ implies that the desire to further increase the level $D$ of democratic participation drops with its actual level. The slope at the inflection point $\sigma(1)=1$ is $-\beta / 2$, viz proportional to the sensibility parameter $\beta$. The time scale for opinion dynamics is hence of the order of $2 T_{V} / \beta$. Alternatively one may interpret the slope and hence $\beta$ as a proxy for the rigor of political correctness.

neural circuitry of the brain $[22,23]$. We have chosen here for simplicity a rescaled Fermi function,

$$
\sigma(D)=\frac{2}{1+\exp (\beta(D-1))},
$$

in physics jargon, for $\sigma(D)$, as illustrated in Figure 1. At the inflection point $D=1$ we have $\sigma(D=1)=1$. The parameter $\beta$, which would correspond to the inverse temperature in physics, is a sensibility parameter, setting the slope $d \sigma / d D=-\beta / 2$ at the inflection point $D=1$.

The evolution equations for $D(t)$ and $V(t)$, equations (1) and (2), have been defined such that the common fixed point $(D, V)=(1,1)$ remains unchanged for all parameter settings. This implies, that (1) and (2) describe the time evolution of quantities which are relative and not bare measures. The steady-state fixed point would evolve on the other side if $D$ and $V$ had been measured in absolute terms [17] and not, as done here, relatively. The renormalization of the steady state to $(1,1)$ does hence encompass the secular backdrop of declining democratic values [21].

\section{Simulations results}

For the parameters entering the evolution equations for the state of the democracy and for the values of the electorate, (1) and (2) respectively, we take $T_{D}=4$ years for the typical adaption time of political actors and $T_{V}=15$ years for the decay time of political values [21]. We start with an overview of the properties of our model, (1) together with (2), for which we set the time delay to $T=4$ years. Alternative values for $T$ will be considered subsequently together with distinct ways to incorporate multiple time delays. For the numerical simulations we discretized the evolution equations (1) and (2), taking one month $(\Delta t=1 / 12$ years $)$ as a basic time step. The such obtained results do not depend qualitatively on the exact value of $\Delta t$. 

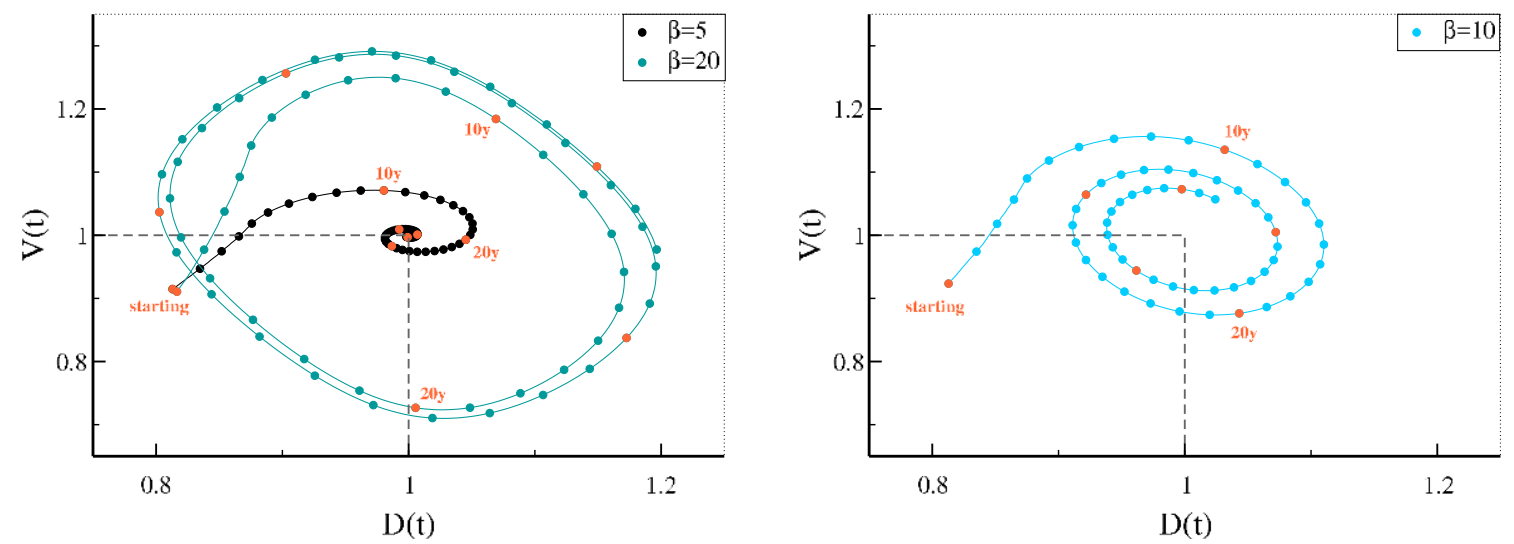

Fig. 2. The result of numerically simulating (1) and (2) for $T=4, T_{D}=4$ and $T_{V}=15$ (years). The system starts (as denoted by the label "starting") right after the initial function, defined for $t \in[-T, 0]$, ends, with every filled point denoting one year (decades are red). Note, that trajectories may intersects themselves for dynamical systems with time delays, as it happens for $\beta=20$. The fixed point $(D, V)=(1,1)$ is stable for $\beta<\beta_{c} \approx 11.36$.

The solution of a time-delayed systems is generically contingent on the choice of the initial function $(D(t), V(t))$, where $t \in[-T, 0][2,24]$. We find, however, that the system (1) and (2) is robust in the sense that the long-time state convergences in all cases to the identical attracting set, which may be either a fixed point or a limit cycle, even when fully random initial functions are selected.

In Figure 2 we present typical trajectories for $\beta=$ $5,10,20$, where the starting function was $(D(t), V(t))=$ $(0.8,0.9)$, with $t \in[-T: 0]$, together with a random jitter $\Delta D=\Delta V=0.02$. The system is stable, as expected, for small values of $\beta$, with the state $(D(t), V(t))$ of the system spiraling toward the fixed point $(1,1)$. The overall time-scale for the evolution is about two decades, as consequence of $T_{V}=15$ years.

For an advanced democracy, characterized by a high sensibility $\beta=20$ to deviations from the political standard, the overall attracting set is a limit cycle with a period of about 24.5 years and an average deviation

$$
D_{F}=\left\langle\sqrt{(D(t)-1)^{2}+(V(t)-1)^{2}}\right\rangle \approx 0.24
$$

from the fixed point $(1,1)$, with the brackets $\langle\cdots\rangle$ denoting the time average. In order to decide whether the limit cycle is far away from the original fixed point, or close, we may compare above value for $D_{F}$ with the functional dependency of the response function $\sigma(D)$ entering (2), as illustrated in Figure 1. We observe, that $D=0.8$ or $D=1.2$ leads to responses $\sigma(D)$ which are exponentially close to 1 and 0 respectively. This implies, that the limit cycle observed for $\beta=20$ in Figure 2 is close to the maximal possible periodic solution supported by (1) and (2). Even for a very large $\beta=80$, to give an example, we find only a slightly increased $D_{F}=0.27$.

Also shown in Figure 2 is a trajectory for $\beta=10$, which spirals in the end into the fixed point $(D, V)=(1,1)$. The extraordinary long time scale needed to reach the equilibrium state, for $\beta=10$, is a consequence of the critical slowing down close to a phase transition, which occurs here at $\beta_{c} \approx 11.36$ (see Sect. 5). It may hence be difficult to distinguish real-world political systems which are subcritical, but close to an instability, from systems which are already unstable.

Our basic presumption is here, that advances in communication and organizational structures lead to a progressing optimization of our societies which is inevitably accompanied with a decreasing tolerance of non-standard behaviors and hence with an increasing $\beta$, as entering (2). In Figure 3 we present a scenario simulation for a timevarying $\beta$, which is held constant at $\beta=5$ for the first ten years, at $\beta=10$ for the subsequent twenty years and at $\beta=20$ thereafter. The system tries initially to reach the equilibrium state $(D, V)=(1,1)$, being subcritical for the first thirty years, with the relaxation toward the fixed point slowing down dramatically when $\beta \rightarrow 10$ (compare Fig. 2). Twenty years at $\beta=10$ are not enough to equilibrate and the final increase to $\beta=20$ leads therefore straightaway to limit-cycle oscillations.

\subsection{Diverging recovery times close to the Hopf bifurcation}

Normal forms allow to classify the type of bifurcations occurring in normal dynamical systems, viz in dynamical systems without time delays [2]. The transition observed here at $\beta_{c} \approx 11.36$ is in this context akin to a classical supercritical Hopf bifurcation, involving a bifurcation from a stable node (fixed point) to a continuously expanding periodic orbit (stable limit cycle) [25].

In order to corroborate this statement we have evaluated the time dependent distance $D_{F}(t)$ of the trajectory from the fixed point, as well as its long time average (4). It is evident from Figure 4, that the size of the final limit cycle shrinks continuously when $\beta$ approaches $\beta_{c}$ from above, as expected for a second-order transition.

It is of interest to examine, for subcritical $\beta<\beta_{c}$, the time scale $T_{\lambda}$ needed to close in to the equilibrium state $(D, V)=(1,1)$, which is given by the inverse of the largest 

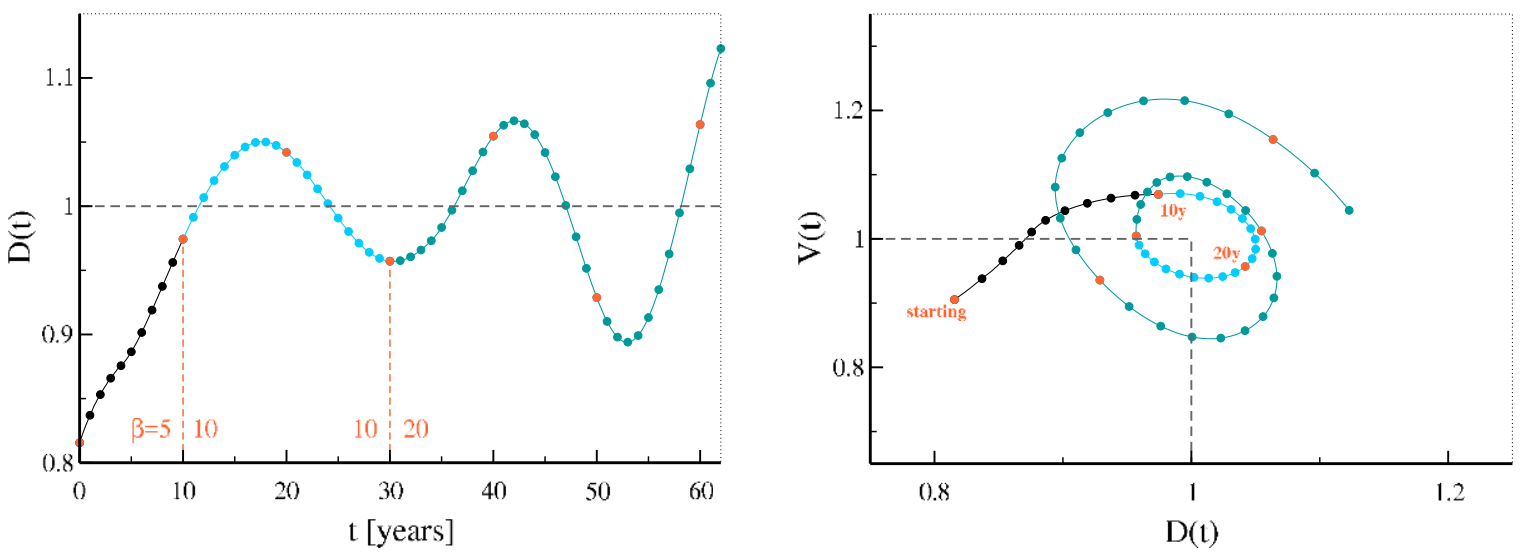

Fig. 3. The result of a numerical experiment, for $T=4, T_{D}=4$ and $T_{V}=15$ (years), where $\beta=5$ for the first 10 years, $\beta=10$ for $t \in[10,30]$ and $\beta=20$ thereafter. The evolution is shown for $D(t)$ as a function of time $($ left $)$ and for $(D, V)$ in state space (right). While still subcritical for $\beta=10$, the relaxation process slows down dramatically due to the closeness to the phase transition occurring at $\beta_{c} \approx 11.36$, compare Figure 2 .

Lyapunov exponent of the fixed point [26]. In Figure 4 we present alternatively the results of a numerical experiment simulating the recovery from an external shock. For a single trajectory, with starting conditions as for Figure 2, the displacement $D_{F}(t)$ from the steady state has been evaluated and fitted by $\exp \left(-t / T_{\lambda}\right)$. We notice that the time needed to recover from the initial displacement becomes of the order of three decades already for $\beta \approx 7.5$, which is still substantially below the critical $\beta_{c} \approx 11.36$. The system is hence very slow to recover from external events pushing it away from the fixed point.

\subsection{Mixture of time delays}

With (1) we assumed that the state $D(t)$ tries to align itself to values the electorate expressed exactly $T$ years before. A mixture of time delays may contribute in reality. We consider with

$$
T_{D} \frac{d}{d t} D(t)=\bar{V}_{\alpha}(t)-D(t)
$$

the coupling of $D(t)$ to two specific distributions $\alpha=1,2$ of lag times,

$$
\begin{aligned}
& \bar{V}_{1}(t)=\frac{1}{2 T} \int_{0}^{2 T} V(t-\tau) d \tau, \\
& \bar{V}_{2}(t)=\frac{1}{T} \int_{0}^{\infty} \mathrm{e}^{-\tau / T} V(t-\tau) d \tau,
\end{aligned}
$$

where $V_{1}(t)$ and $V_{2}(t)$ correspond respectively to a flat distribution, with $T \in[0,2 T]$, and to exponentially discounted delay times. The average time delay stays at $T$ in both cases. We find, as shown in Figure 4, that a flat distribution, viz $\bar{V}_{1}$ in equation (5), induces only relative minor quantitative changes, with all qualitative features of the original model (1) remaining untouched. There is a slight upward renormalization, when using $\bar{V}_{1}$, of the critical sensitivity from $\beta_{c} \approx 11.36$, as obtained for (1), to $\beta_{c} \approx 13.5$.
For exponentially discounted lag times, describing the common but not exclusive case that past messages are progressively discounted in the context of political communication [27], we find numerically that $\beta_{c} \approx 23.7$, which is now substantially increased, but otherwise no overall qualitative changes.

\section{Stability analysis}

The stability of the fixed point $(D, V)=(1,1)$ can be examined [28] by linearizing the evolution equations (1) and (2)

$$
\begin{aligned}
& T_{D} \frac{d}{d t} \delta D(t)=\delta V(t-T)-\delta D(t), \\
& T_{V} \frac{d}{d t} \delta V(t)=-\frac{\beta}{2} \delta D(t)-\delta V(t),
\end{aligned}
$$

where $\delta D=D-1$ and $\delta V=V-1$. The Ansatz $\delta D(t)=$ $D_{0} \exp (\lambda t)$ and $\delta V(t)=V_{0} \exp (\lambda t)$ leads to

$$
V_{0} \mathrm{e}^{-\lambda T}=D_{0}\left(1+T_{D} \lambda\right), \quad D_{0}=-\frac{2 V_{0}}{\beta}\left(1+T_{V} \lambda\right),
$$

and hence to

$$
\mathrm{e}^{-\lambda T}=-\frac{2}{\beta}\left(1+T_{V} \lambda\right)\left(1+T_{D} \lambda\right) .
$$

The Lyapunov exponent $\lambda=\lambda^{\prime}+i \lambda^{\prime \prime}$ is generically complex, becoming purely imaginary, with $\lambda^{\prime}=0$, at the bifurcation $\beta \rightarrow \beta_{c}$. The real and imaginary components of (10) then are:

$$
\begin{aligned}
& \cos \left(\lambda^{\prime \prime} T\right)=-\frac{2}{\beta_{c}}\left(1-T_{V} T_{D}\left(\lambda^{\prime \prime}\right)^{2}\right), \\
& \sin \left(\lambda^{\prime \prime} T\right)=\frac{2}{\beta_{c}}\left(T_{V}+T_{D}\right) \lambda^{\prime \prime},
\end{aligned}
$$



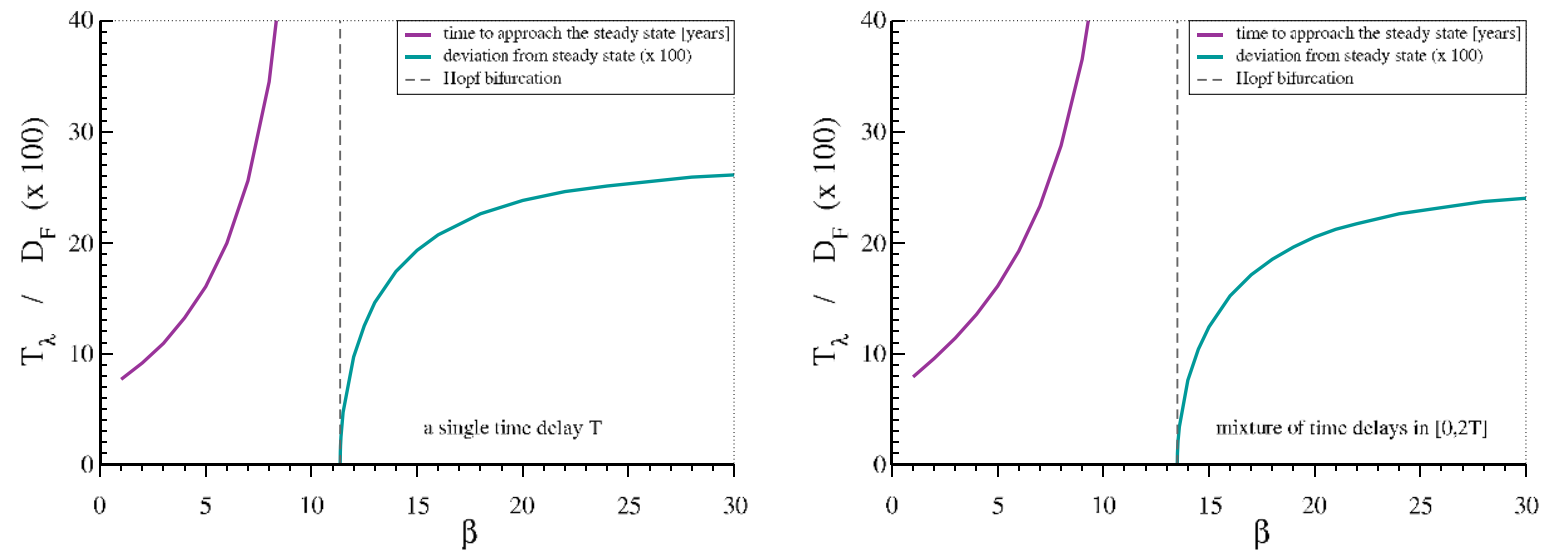

Fig. 4. The results of evaluating the Euclidean distance $D_{F}$ from the fixed point $(D, V)=(1,1)$. For $\beta>\beta_{c}$ (dashed vertical line) the time-average $D_{F}$, equation (4), of the limit cycle is shown (multiplied by 100). For $\beta<\beta_{c}$ the relaxation time $T_{\lambda}$ is shown (in years). $T_{\lambda}$, which is also the time needed to recover from external shocks, has been obtained by fitting the timedependent Euclidean distance $D_{F}=D_{F}(t)$ by $\exp \left(-t / T_{\lambda}\right)$. The data is for the model with a single time delay $T=4($ Eq. $(1)$, left panel) and for the model with a uniform mixture of time delays (Eq. (5), right panel) and otherwise identical parameters. The respective critical sensitivities are $\beta_{c} \approx 11.36$ (left) and $\beta_{c} \approx 13.5$ (right).

or

$$
\tan \left(T \lambda^{\prime \prime}\right)=\frac{\left(T_{D}+T_{V}\right) \lambda^{\prime \prime}}{T_{D} T_{V}\left(\lambda^{\prime \prime}\right)^{2}-1}
$$

and

$$
\frac{\beta_{c}^{2}}{4}=\left(1+\left(T_{D} \lambda^{\prime \prime}\right)^{2}\right)\left(1+\left(T_{V} \lambda^{\prime \prime}\right)^{2}\right)
$$

where we have used that

$$
\begin{aligned}
\left(T_{D} T_{V}\left(\lambda^{\prime \prime}\right)^{2}-1\right)^{2} & +\left(T_{D}+T_{V}\right)^{2}\left(\lambda^{\prime \prime}\right)^{2} \\
& =\left(1+\left(T_{D} \lambda^{\prime \prime}\right)^{2}\right)\left(1+\left(T_{V} \lambda^{\prime \prime}\right)^{2}\right)
\end{aligned}
$$

One solves first (13) for $\lambda^{\prime \prime}$ and then (14) for $\beta_{c}$. The corresponding phase diagram is presented in Figure 5 for fixed $T_{D}=4$ and $T_{V}=15$. The locus of the phase transition at $T=4$ is $\beta_{c}=11.4$, which differs only marginally from the one found in the numerical simulation, $\beta_{c}=11.36$, for which time had been discretized (using $\Delta t=1 / 12$ ).

\subsection{Uniform mixture of time delays}

For the case (5) of an uniform mixture of time delays one replaces $\exp (-\lambda T)$ in (10) by $\int \exp (-\lambda \tau) d \tau /(2 T)$, obtaining

$$
\begin{aligned}
\frac{1}{2 T} \int_{0}^{2 T} \cos \left(\lambda^{\prime \prime} \tau\right) d \tau & =\frac{2}{\beta_{c}}\left[T_{V} T_{D}\left(\lambda^{\prime \prime}\right)^{2}-1\right] \\
\frac{1}{2 T} \int_{0}^{2 T} \sin \left(\lambda^{\prime \prime} \tau\right) d \tau & =\frac{2}{\beta_{c}}\left(T_{V}+T_{D}\right) \lambda^{\prime \prime}
\end{aligned}
$$

which results in turn, after carrying out the respective integrals, in

$$
\begin{aligned}
\sin \left(2 T \lambda^{\prime \prime}\right) & =\frac{4 T \lambda^{\prime \prime}}{\beta_{c}}\left[T_{V} T_{D}\left(\lambda^{\prime \prime}\right)^{2}-1\right], \\
1-\cos \left(2 T \lambda^{\prime \prime}\right) & =\frac{4 T \lambda^{\prime \prime}}{\beta_{c}}\left(T_{V}+T_{D}\right) \lambda^{\prime \prime} .
\end{aligned}
$$

With $\sin \left(2 \lambda^{\prime \prime}\right)=2 \sin \left(T \lambda^{\prime \prime}\right) \cos \left(T \lambda^{\prime \prime}\right)$ and $\cos \left(2 T \lambda^{\prime \prime}\right)=$ $1-2 \sin ^{2}\left(T \lambda^{\prime \prime}\right)$ we then obtain

$$
\tan \left(T \lambda^{\prime \prime}\right)=\frac{\left(T_{D}+T_{V}\right) \lambda^{\prime \prime}}{T_{D} T_{V}\left(\lambda^{\prime \prime}\right)^{2}-1}
$$

and

$$
\frac{\beta_{c}}{2} \frac{T_{D}+T_{V}}{T}=\left(1+\left(T_{D} \lambda^{\prime \prime}\right)^{2}\right)\left(1+\left(T_{V} \lambda^{\prime \prime}\right)^{2}\right) .
$$

Note that the expressions (15) and (13) for the imaginary component $\lambda^{\prime \prime}$ of the Lyapunov exponents are identical and, correspondingly, also the right-hand sides of (16) and (14). For above transformations we used

$$
\left[1-\cos \left(2 T \lambda^{\prime \prime}\right)\right] / \sin \left(2 T \lambda^{\prime \prime}\right)=\tan \left(T \lambda^{\prime \prime}\right)
$$

and that

$$
\begin{aligned}
4 \sin ^{2}\left(T \lambda^{\prime \prime}\right) & =\frac{\left(4 T \lambda^{\prime \prime}\right)^{2}}{\beta_{c}^{2}}\left(1+\left(T_{D} \lambda^{\prime \prime}\right)^{2}\right)\left(1+\left(T_{V} \lambda^{\prime \prime}\right)^{2}\right) \\
& =\frac{4 \tan ^{2}\left(T \lambda^{\prime \prime}\right)}{1+\tan ^{2}\left(T \lambda^{\prime \prime}\right)}
\end{aligned}
$$

can be simplified when using (15). The bifurcation line resulting from (16), which has been included in Figure 5, runs somewhat parallel to the one obtained via (14) for the case of a single delay time, closing in for $T \ll T_{V}$, when 

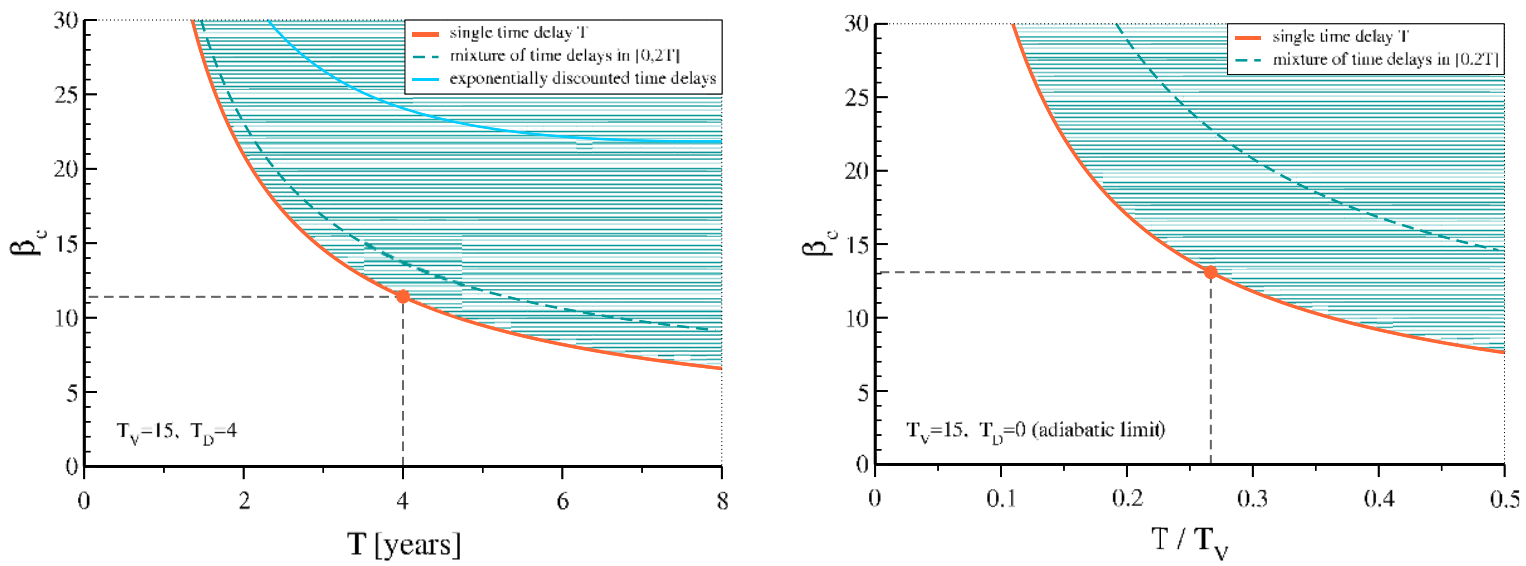

Fig. 5. The Hopf bifurcation line for the case of a single time delay (full red curve), for a uniform distribution of delay times (dashed cyan curve) and for exponentially distributed time delays (full blue curve). The attracting state is a limit cycle above the respective lines (viz in the shaded region for the case of a single time delay), and a fixed point otherwise. The dashed rectangle indicates the case of a single time delay $T=4$, and an adaption time scale for $V(t)$ of $T_{V}=15$. Left: For $T_{D}=4$ and the original model (1) and (2), respectively (5), for which the bifurcations lines $\beta_{c}$ are determined by (14), (16) and (19). Right: For the adiabatic limit (20), obtained when $T_{D} \rightarrow 0$. In this limit there is no Hopf bifurcation for exponentially discounted time delays.

the actual distribution of lag times becomes unimportant. For $T=4$ we find that $\beta_{c}$ increases from $\beta_{c}=11.4$ to $\beta_{c}=13.68$.

Comparing (13) and (15) one finds, remarkably, that the imaginary part $\lambda^{\prime \prime}$ of the Lyapunov exponent is identical at criticality, albeit at different values of $\beta_{c}$. This implies, that the revolution frequencies of the resulting limit cycles are identical in the respective limits $\beta \rightarrow \beta_{c}$ from above.

\subsection{Exponentially discounted time delays}

For exponentially discounted delay times (5) we need

$$
\begin{aligned}
& \frac{1}{T} \int_{0}^{2 T} \mathrm{e}^{-t / T} \cos \left(\lambda^{\prime \prime} \tau\right) d \tau=\frac{1}{1+\left(T \lambda^{\prime \prime}\right)^{2}}, \\
& \frac{1}{T} \int_{0}^{2 T} \mathrm{e}^{-t / T} \sin \left(\lambda^{\prime \prime} \tau\right) d \tau=\frac{T \lambda^{\prime \prime}}{1+\left(T \lambda^{\prime \prime}\right)^{2}},
\end{aligned}
$$

which results respectively in

$$
\begin{aligned}
& \frac{1}{1+\left(T \lambda^{\prime \prime}\right)^{2}}=\frac{2}{\beta_{c}}\left(T_{V} T_{D}\left(\lambda^{\prime \prime}\right)^{2}-1\right), \\
& \frac{1}{1+\left(T \lambda^{\prime \prime}\right)^{2}}=\frac{2}{\beta_{c}} \frac{T_{V}+T_{D}}{T}
\end{aligned}
$$

instead of (11) and (12). We then find

$$
\begin{aligned}
\left(\lambda^{\prime \prime}\right)^{2} & =\frac{T_{D}+T_{V}+T}{T_{D} T_{V} T} \\
\beta_{c} & =2 \frac{T_{D}+T_{V}}{T}\left[1+\left(T \lambda^{\prime \prime}\right)^{2}\right]
\end{aligned}
$$

for the Hopf bifurcation line. The critical $\beta_{c}$ has been included in Figure 5. For $T_{D}=4=T$ and $T_{V}=15$ the resulting $\beta_{c}=24.1$ is again marginally larger than the value, $\beta_{c} \approx 23.7$, obtained from corresponding time discretized numerical simulation.

\subsection{Adiabatic limit}

We have shown above that our model is robust against changes in the distribution of time delays. The nature of the attracting states are also not sensitively dependent on the ratio of $T_{D} / T_{V}$. It is illustrative, in this context, to examine the adiabatic limit $T_{D} \ll T_{V}$ of (1) and (2), for which $D(t)$ follows closely $V(t-T)$. In this case one can substitute $D(t)$ by $V(t-T)$ in (2), obtaining

$$
T_{V} \frac{d}{d t} V(t)=\sigma(V(t-T))-V(t)
$$

The locus of the bifurcation is determined by (14) in the limit $T_{D} \rightarrow 0$, or, alternatively, by

$$
\tan \left(x T / T_{V}\right)=-x, \frac{\beta_{c}^{2}}{4}=1+x^{2}, x=T_{V} \lambda^{\prime \prime}
$$

when using rescaled variables. $\beta_{c}$ is then dependent only on the ratio $T / T_{V}$, as shown in Figure 5 . For the case of a uniform mixture of time delays (15) and (16) reduce to

$$
\tan \left(x T / T_{V}\right)=-x, \quad \frac{\beta_{c}}{2} \frac{T_{V}}{T}=1+x^{2},
$$

in the limit $T_{D} \rightarrow 0$. One notices, compare Figure 5 , that there is a substantial quantitative difference in the adiabatic limit between having a single and a mixture of time delays.

Interestingly, there is no phase transition in the adiabatic limit for the case of exponentially discounted time delays, with (17) having no solution in the limit $T_{D} \rightarrow 0$. 


\subsection{Properties of the phase diagram}

The phase diagrams presented in Figure 5 have a series of common features.

- The Hopf bifurcation line is a monotonically decreasing function. For small time delays $T$ one needs a higher sensibility $\beta>\beta_{c}$ for the instability to occur, and vice verse.

- There is no minimal time delay $T$, viz there is a critical $\beta_{c}<\infty$ for any $T>0$, with

$$
\lim _{T / T_{V} \rightarrow 0} \beta_{c}\left(T / T_{V}\right) \rightarrow \infty
$$

The fixed point is hence stable for all $\beta$ when there is no time delay, $T=0$.

- There is a lower $\beta_{c}$ below which the fixed point is stable even when $T$ is arbitrary large. In the adiabatic limit (21) one needs $\beta_{c}>2$.

- The imaginary part $\lambda^{\prime \prime}$ of the Lyapunov exponent needs to be non-zero for (13) and (15) to have a nontrivial solution. $\lambda^{\prime \prime}$ is hence finite at the transition, the tell-sign of a Hopf bifurcation [2]. The revolution frequency of the limit cycle, which is of the order of $1 /\left|\lambda^{\prime \prime}\right|$, is hence not critical, varying smoothly above the transition.

In the vicinity of the transition the sensibility $\beta$ induces a speed-up of the reactive value dynamics, as evident from the linearized equations (8) and (9), by a factor $\beta / 2$, which may be identified with a corresponding acceleration of opinion dynamics. The overall time needed to reach the fixed point nevertheless diverges as $1 / \lambda^{\prime} \sim 1 /\left|\beta-\beta_{c}\right|$. This phenomenon, known as critical slowing down, is observed generically in dynamical systems close to a tipping point. It is observed in a wide range of settings, affecting, e.g., the resilience of ecosystems [29] as well as the evolution of the climate prior to a major shift [30]. The increased time scales needed to react to disturbances close the instability are also evident in Figure 4.

\section{Discussion}

There are two mutually not exclusive routes to describe the conflict between slow political decision making and accelerating social dynamics $[6,8,10]$. In the first view politics continuously adapts, over the course of $T_{D}$ years, to the current demands of the electorate. Time lags are absent in this scenario and the system stable for all parameters. Politics then evolves around a stable state, with deviations from the fixed point driven exclusively by external events.

Here we have examined a second possibility, namely that a certain fraction of political decision making results from the response to demands the electorate voiced $T$ years ago. The time delay $T$ may be either fixed or drawn from a continuous distribution, as described by equations (1) and (5) respectively. For both cases we find that the socio-political system becomes inherently unstable whenever the electorate responds sensitively to political changes. This conclusion, which is robust and independent of the details of the here used model, results from the fact that time delays will inherently amplify fluctuations once their influence becomes substantial.

In our model the sensitivity $\beta$ of the electorate leads to typical reaction times $2 T_{V} / \beta$, as evident form the linearized evolution equation (9), where $T_{V}$ is the time scale for the long-term evolution of basic political values. In order to obtain estimates for real-world political communication we considered the case of exponentially discounted time delays, for which the instability occurs at $\beta_{c} \approx 24.1$ for $T=4$ and at $\beta_{c} \approx 50.7$ for $T=1$ (compare Fig. 5). Socio-political instabilities then start to manifest themselves for $T=4$ when the corresponding times scale $2 T_{V} / \beta_{c}$ for the opinion dynamics falls below $30 / 24.1$ years (about 15 months). For a time delay of one year, $T=1$, instabilities develop when the opinion dynamics takes place on time scale below 30/50.7 years (about 7 months).

Our estimates for the tipping point of political opinion dynamics, 7-15 months when assuming mean time delays of the order of 1-4 years, are for aggregate processes which include the effects of fast news propagation as well as the consequences of slowly but continuously changing preset political beliefs. It is conceivable within out model that western democracies have seen the unfolding of a slow but steady long-term acceleration of opinion dynamics, with the passing of the threshold of 7-15 months contributing to the recent emergence of political styles disrupting political conventions considered hitherto as fundamental [20]. External effects, such as the 2007-08 financial crisis $[31,32]$, would induce in this view an additional temporary but sharp rise in $\beta$.

An important aspect regards the time needed to recover from an external disrupting event, such as a global crisis. Naively one may expect that the accelerating pace of opinion formation observed in advanced democracies would reduce typical recovery times. The contrary is however the case. It is well known, as illustrated in Figure 4, that second order instabilities lead to critical slowing down in their proximity and hence to diverging recovery times. As a consequence one observes long-lasting oscillations even below the actual transition, as illustrated in Figure 2. Analogous oscillations matching both the period (about 20 years), and the magnitude (10-15\%), have be observed since the early 1990s in Australian polls studying aggregate value orientations along the materialism vs. postmaterialism axis [33]. A substantially larger corpus of data would however been needed for an eventual validation, or falsification, of the here presented approach. Note that our framework describes instabilities arising within representative democracies and not transitions to non-democratic regimes.

The scope of the work presented here is to point out a phenomenon of possible key importance for the understanding of the long-term stability of representative democracies. The instabilities we find lead to oscillatory but not to irregular socio-political states. One possibility to extend our study would however be to consider time delays varying periodically with the election cycle. It is to be expected that such kinds of non-constant time 
delays would act as periodic drivings [34], which are in turn known to induce transitions to chaotic states in nonlinear dynamical systems. We note in this context that transitions to potentially disrupting states with runaway opinion growth have been observed [35] in agent based simulations examining the response of an electorate to rising levels of immigration.

We thank Karolin Kappler regarding discussions concerning social acceleration, Daniel Lambach regarding time delays in democratic structures and Roser Valenti for reading the manuscript.

\section{Author contribution statement}

The author is responsible for all content.

Open Access This is an open access article distributed under the terms of the Creative Commons Attribution License (http://creativecommons.org/licenses/by/4.0), which permits unrestricted use, distribution, and reproduction in any medium, provided the original work is properly cited.

\section{References}

1. T. Erneux, Applied delay differential equations (Springer Science \& Business Media, 2009)

2. C. Gros, Complex and adaptive dynamical systems: a primer (Springer, 2015)

3. G. Singh, I.S. Ahuja, Int. J. Bus. Contin. Risk Manag. 3, $57(2012)$

4. J. Schnellenbach, C. Schubert, Eur. J. Polit. Econ. 40, 395 (2015)

5. S.S. Wolin, Theory Event 1, 1 (1997)

6. K.H. Goetz, West Eur. Polit. 37, 379 (2014)

7. J. Fleischer, Public Manag. Rev. 15, 313 (2013)

8. M. Tomba, South Atl. Q. 113, 353 (2014)

9. M. Wolffsohn, T. Brechenmacher, Int. J. Public Opin. Res. 13, $116(2001)$

10. H. Rosa, Social acceleration: a new theory of modernity (Columbia University Press, 2013)

11. E. Pain, Science 356, 14 (2017)

12. A. Menon, J.-P. Salter, Int. Aff. 92, 1297 (2016)
13. R.J. Dalton, S.E. Scarrow, B.E. Cain, J. Democr. 15, 124 (2004)

14. G. Hughes, Political correctness: a history of semantics and culture (John Wiley \& Sons, 2011)

15. A. Maass, C. Suitner, E. Merkel, Does political correctness make (social) sense? in Social Cognition and communication, edited by J.P. Forgas, O. Vincze, J. Laszlo (Psychology Press, New York, NY, 2013), pp. 331-346

16. M.A. Abdollahian, T.G. Coan, H. Oh, B.A. Yesilada, Int. Stud. Q. 56, 827 (2012)

17. V. Spaiser, S. Ranganathan, R.P. Mann, D.J.T. Sumpter, PLOS ONE 9, e97856 (2014)

18. A.C. Alexander, R. Inglehart, C. Welzel, Int. Polit. Sci. Rev. 33, 41 (2012)

19. S. Ranganathan, V. Spaiser, R.P. Mann, D.J.T. Sumpter, PLOS ONE 9, e86468 (2014)

20. R. Inglehart, P. Norris, Trump, Brexit, and the rise of populism: economic have-nots and cultural backlash, HKS Faculty Research Working Paper Series RWP16-026, 2016

21. R.S. Foa, Y. Mounk, J. Democr. 27, 5 (2016)

22. S. Dehaene, Trends Cogn. Sci. 7, 145 (2003)

23. C. Gros, G. Kaczor, D. Marković, Eur. Phys. J. B 85, 28 (2012)

24. J.-P. Richard, Automatica 39, 1667 (2003)

25. M.J. Piotrowska, U. Foryś, Math. Comput. Model. 54, 2183 (2011)

26. H. Wernecke, B. Sándor, C. Gros, Sci. Rep. 7, 1087 (2017)

27. D. Chong, J.N. Druckman, Am. Polit. Sci. Rev. 104, 663 (2010)

28. E. Boukas, Z. Liu, Deterministic and stochastic time-delay systems (Springer Science \& Business Media, 2012)

29. E.H. Van Nes, M. Scheffer, Am. Nat. 169, 738 (2007)

30. V. Dakos, M. Scheffer, E.H. van Nes, V. Brovkin, V. Petoukhov, H. Held, Proc. Natl. Acad. Sci. 105, 14308 (2008)

31. R.J. Shiller, The subprime solution: how today's global financial crisis happened, and what to do about it (Princeton University Press, 2012)

32. M. Funke, M. Schularick, C. Trebesch, Eur. Econ. Rev. 88, 227 (2016)

33. B. Tranter, Sage Open 5 (2015), DOI: $10.1177 / 2158244015591826$

34. D. d'Humieres, M.R. Beasley, B.A. Huberman, A. Libchaber, Phys. Rev. A 26, 3483 (1982)

35. B. Podobnik, M. Jusup, D. Kovac, H.E. Stanley, Complexity 2017, 1580526 (2017) 